\title{
Limitations of VCG-Based Mechanisms
}

\author{
Shahar Dobzinski Noam Nisan *
}

November 20, 2006

\begin{abstract}
We consider computationally-efficient incentive-compatible mechanisms that use the VCG payment scheme, and study how well they can approximate the social welfare in auction settings. We present a novel technique for setting lower bounds on the approximation ratio of this type of mechanisms. Specifically, for combinatorial auctions among submodular (and thus also subadditive) bidders we prove an $\Omega\left(m^{\frac{1}{6}}\right)$ lower bound, which is close to the known upper bound of $O\left(m^{\frac{1}{2}}\right)$, and qualitatively higher than the constant factor approximation possible from a purely computational point of view.
\end{abstract}

\section{Introduction}

\section{$1.1 \quad$ Background}

Algorithmic Mechanism design attempts to design protocols for distributed environments, such as the Internet, where the different participants each have their own selfish goals and are assumed to rationally attempt optimizing their own goals rather than just follow any prescribed protocol. The key target in this area is the design of incentive-compatible mechanisms - also called truthful or strategy-proof mechanisms - whose payment schemes motivate the participants to correctly report their private information ${ }^{1}$. For a general introduction to the economic field of mechanism design see [21] and for an introduction to algorithmic mechanism design and further motivation see [25].

Typical problems in this setting involve allocation of various resources and a paradigmatic abstraction is that of combinatorial auctions. In this problem $m$ heterogenous "items" need to be allocated between $n$ "bidders". Each bidder $i$ holds a valuation function $v_{i}$ that specifies for each subset of the items $S \subseteq\{1 \ldots m\}$ the bidder's value $v_{i}(S)$ from winning the "bundle" $S$. The challenge is to find a partition $S_{1} \ldots S_{n}$ of the items that maximizes the social welfare $\Sigma_{i} v_{i}\left(S_{i}\right)$. This problem presents a combination of algorithmic difficulty (it is NP-complete), representational difficulty (the valuation functions are exponential size objects) and strategic difficulty (ensuring incentive compatibility).

The key positive technique for achieving incentive compatibility is that of VCG mechanisms $[29,6,13]$ : if player $i$ 's value from the chosen algorithmic outcome $a$ is $v_{i}(a)$, then we charge player $i$ the quantity $h_{i}\left(v_{-i}\right)-\Sigma_{j \neq i} v_{j}(a)$, where $h_{i}$ is an arbitrary fixed function that does not depend on

*The School of Computer Science and Engineering, The Hebrew University of Jerusalem, \{shahard, noam\}@cs.huji.ac.il. Supported by grants from the Israel Science Foundation and the USA-Israel Bi-national Science Foundation.

${ }^{1}$ This paper - as most previous work in algorithmic mechanism design - considers the simple and strong notion of incentive compatibility in dominant strategies. This covers also protocols where the desired results are obtained as arbitrary dominant-strategy equilibria, as the revelation principle allows converting such mechanisms to incentive compatible ones. 
$v_{i}$. A powerful observation is that if the algorithmic outcome $a$ always maximizes the social welfare, $\Sigma_{i} v_{i}(a)$, then the VCG payment rule results in an incentive compatible mechanism. However, in most interesting computational scenarios, including combinatorial auctions, achieving exact optima is computationally intractable, and one must settle for heuristics or approximations. A key clash between the strategic and algorithmic considerations is that once only approximations or heuristics are chosen, the VCG payment rule no longer leads to incentive compatibility. See, e.g., [20, 24].

The heart of "algorithmic mechanism design" is trying to overcome this clash: design mechanisms that are both computationally tractable (and thus only approximate the optimum) and strategically incentive compatible. The key question is always to what extent do the strategic requirements degrade the quality of the solution beyond the degradation implied by the purely computational constraints. At this point one can summarize the state of the art as having clear characterizations and many good upper bounds (approximation mechanisms) for various classes of "single parameter" problems (e.g. [20, 22, 1]). On the other hand, for more general "multi-parameter" problems like combinatorial auctions, one can safely say that - almost - no progress has been made in obtaining upper bounds or lower bounds. In this paper we wish to address exactly this issue, attempting to prove lower bounds - showing that the strategic constraints do impose an additional burden beyond the computational ones.

\section{$1.2 \quad$ VCG-based mechanisms}

We are unable to prove general lower bounds, so we limit ourselves to a natural - and interesting in itself - class of mechanisms: VCG-based ones. These are the ones that use the same payment idea as in VCG: each bidder $i$ pays $h_{i}\left(v_{-i}\right)-\Sigma_{j \neq i} v_{j}(a)$, where $a$ is the algorithmic output). This is a rather natural choice given the complete lack of other techniques for multi-parameter settings. This class was studied in [24], who observed that the following family of allocation algorithms do yield incentive-compatible VCG-based mechanisms:

Definition: An allocation algorithm (that produces an output $a \in \mathcal{A}$ for each input $v_{1} \ldots v_{n}$, where $\mathcal{A}$ is the set of possible alternatives) is called "maximal-in-range" (henceforth MIR) if it completely optimizes the social welfare over some subrange $\mathcal{R} \subseteq \mathcal{A}$. I.e., for some $\mathcal{R} \subseteq \mathcal{A}$, we have that for all $v_{1} \ldots v_{n}, a \in \arg \max _{a \in \mathcal{R}} \Sigma_{i} v_{i}(a)$.

I.e., MIR algorithms use the following natural and simple strategy to find an approximately optimal solution: they just optimally search within a pre-specified sub-range of feasible solutions - a subrange over which optimal search is algorithmically feasible. An example for such a strategy, in a different context, is approximating the optimal Steiner tree by taking the best spanning tree [28]. Another example is Arora's approximation for the traveling salesman problem [2].

The main result of [24] states that this is essentially it - no other VCG-based mechanisms are incentive compatible.

Theorem [24]: The allocation algorithm of any incentive-compatible VCG-based mechanism for combinatorial auctions is equivalent to a maximal-in-range algorithm.

"Equivalent" here means that the social utilities are identical for all inputs, i.e. if $a$ and $b$ are the outputs of the two allocation algorithms for input $v_{1} \ldots v_{n}$ then $\Sigma_{i} v_{i}(a)=\Sigma_{i} v_{i}(b)$. In particular, the outputs must coincide generically - except perhaps in case of ties. In [24] this theorem was presented for two specific types of mechanism design problems, but the result is more general. For completeness, we spell this out in Section 3.

In this paper, we prove bounds on the power of such MIR algorithms, equivalently, for VCG-based mechanisms. Let us explicitly justify why are lower bounds for this restricted class interesting: 
1. Their power: Many incentive compatible mechanisms put forward do fall into this category. This includes a slightly non-trivial $O(m / \sqrt{\log m})$-approximation for general combinatorial auctions [14], an $O(\sqrt{m})$-approximation for combinatorial auctions with complement-free bidders [9], a 2-approximation for multi-unit auctions, which is improved to a PTAS for certain bidding languages [7], welfare maximization in congestion games [5], and several auctions for "geometric figures" on the plane [3].

2. Lack of alternatives: Not only are VCG mechanisms the only general method known for constructing incentive compatible mechanisms in multi-parameter settings, there is just a single additional example of any non-VCG incentive compatible mechanism in any multi-parameter domain [4]. Moreover, Roberts' classic theorem [27] states that in completely unrestricted domains the only incentive compatible mechanisms are weighted versions of VCG-based mechanisms. In [17], it was suggested that Roberts' theorem could be extended to many other domains including combinatorial auctions and multi-unit auctions. In [17], they were only able to prove this for special cases or under additional assumptions, and left the general question open. If this line of attack reaches its conclusion, then our lower bounds would apply in general.

We should note that if randomization is allowed then the second point no longer holds with such force, as several randomized incentive compatible mechanisms are known $[8,18,11]$. It is not known, however, whether any of these can be de-randomized (even if $\mathrm{P}=\mathrm{BPP}$ ). The resolution of this question relies, of course, on the successful characterization of deterministic incentive compatible mechanisms.

\subsection{Our Main Result}

Our main result provides a lower bound for the approximation factor that can be achieved by incentive-compatible VCG-based mechanisms for combinatorial auctions. Our lower bound applies to the subclass of submodular valuations $\left(v_{i}(S \cup T)+v_{i}(S \cap T) \leq v_{i}(S)+V_{i}(T)\right.$ for all $\left.S, T\right)$ and thus also to its superset class of complement-free valuations $\left(v_{i}(S \cup T) \leq v_{i}(S)+v_{i}(T)\right.$ for all $\left.S, T\right)$ - two classes of valuations which have been extensively studied $[19,9,11,10,12,15]$. The best deterministic mechanism for this case is the VCG-based $O(\sqrt{m})$-mechanism of $[9]^{2}$. We note that the technique we present is quite general, and we believe it will turn even more useful by extending our results to other domains as well.

A word about the computational model is in place here: the "inputs" to the mechanism, the $v_{i}$ 's, are exponential sized objects (in the number of items $m$ ), but the mechanisms should run in time polynomial in $n$ and $m$. Thus it is assumed that the mechanism repeatedly queries the bidders. The upper bounds in the table always assume some specific natural type of query (usually a "demand query"), while all lower bounds apply for every type of query and are in fact communication lower bounds.

Theorem: Every VCG-based mechanism for approximating the welfare in combinatorial auctions with submodular bidders that uses a sub-exponential number of queries to the bidders achieves an approximation factor of $\Omega\left(\min \left(n, m^{1 / 6}\right)\right)$.

The proof proceeds by combinatorially analyzing maximum in range allocation algorithms. The analysis shows that if the range is "large" then optimizing over it requires exponential communication, while if it is "small" then it can not achieve a good approximation ratio. It turns out that "large"

\footnotetext{
${ }^{2}$ This is also the best incentive-compatible mechanism for subadditive bidders in general, as the randomized $O\left(\log ^{2} m\right)$-approximation mechanism of [8] does not apply to the class of complement-free bidders.
} 
and "small" in this sense cannot just be interpreted in terms of the size of the range. Instead we define two "complexity measures" of a set of partitions (which is what the range is). One of them, termed the intersection number, is shown to bound from below the communication complexity of optimization over the range. The other, termed the cover number, is shown to bound from above the approximation ratio achieved by allocations in the range. Our main combinatorial lemma, which may be of independent interest, shows that these two complexity measures are related to each other.

We stress that although communication complexity methods were already used in [23, 26] in the context of combinatorial auctions, our methods are completely different and this difference is inherent: $[23,26]$ did not consider incentives issues at all.

\subsection{Open Problems}

Open problems are left at various levels of generality. At the most specific level, the problem is to close the gap between the $m^{1 / 6}$ lower bound and the $m^{1 / 2}$ upper bound. (We can only improve the lower bound to $m^{1 / 5-\epsilon}$.) At the next level, the question is how well can VCG-based mechanisms approximate the social welfare in combinatorial auctions with general valuations? We only have a slightly better lower bound than what we have for the submodular case, but the upper bound of [14] is nearly linear.

Of course, the real questions are always how well can arbitrary computationally efficient incentive compatible mechanism do - not just VCG-based ones - and obtaining any such lower bound would be of great interest. This would likely require some advances in the "LMN-program" [17] of characterizing incentive compatible mechanisms in multi-parameter domains.

\subsection{Paper Structure}

In Section 2 we prove the main theorem. Section 3 brings the characterization of VCG-based mechanisms of [24], generalized for our setting.

\section{Combinatorial Auctions with Submodular Bidders}

\subsection{Combinatorial Auctions: Preliminaries}

In a combinatorial auction we have a set $M,|M|=m$, of heterogeneous items and a set of $N$ bidders, $|N|=n$. Each bidder $i$ has a valuation function $v_{i}: 2^{M} \rightarrow \mathbb{R}$. We assume that each valuation $v_{i}$ is normalized (i.e., $v_{i}(\emptyset)=0$ ) and monotone (for each $S \subseteq T, v_{i}(S) \leq v_{i}(T)$ ). An allocation is an $n$-tuple $S=\left(S_{1}, \ldots, S_{n}\right)$, where for each $i, S_{i} \subseteq M$, and for each $i \neq i^{\prime}, S_{i} \cap S_{i^{\prime}}=\emptyset$. Our goal is to find an allocation $S$ that maximizes the welfare $\Sigma_{i} v_{i}\left(S_{i}\right)$.

A valuation $v$ is said to be submodular if it exhibits decreasing marginal utilities. I.e., for each $S \subseteq T \subseteq M$ and $j \notin S$, we have that $v_{i}(T \cup\{j\})-v_{i}(T) \leq v_{i}(S \cup\{j\})-v_{i}(S)$. We will also use a very simple subset of submodular valuations called additive valuations. A valuation $v$ is said to be additive if for each $S \subseteq M$, we have that $v(S)=\Sigma_{j \in S} v(\{j\})$.

\subsection{The Main Result}

In this section we analyze the power of MIR algorithms in the context of combinatorial auctions with submodular bidders. For this setting, an $O(\sqrt{m})$-approximation MIR algorithm is known [9]. We will show that this is (almost) the best approximation one can get using MIR algorithms. The theorem 
is stated only for MIR algorithms but we will point out how it can be extended to algorithms that are equivalent to MIR algorithms, and thus to all VCG-based mechanisms ${ }^{3}$.

Theorem 2.1 Every MIR mechanism for approximating the welfare in combinatorial auctions with submodular bidders that uses $O\left(e^{\frac{1}{15}}\right)$ bits of communication achieves an approximation factor of $\Omega\left(\min \left(n, m^{1 / 6}\right)\right)$. This result also holds for the randomized and non-deterministic settings.

We define two complexity measures for the range $\mathcal{R}$ of an MIR algorithm $A$ : the cover number, and the intersection number. The cover number roughly corresponds to the size of the range $\mathcal{R}$. We will show, using the probabilistic method, that if the cover number is "small" then there exists an instance such that $A$ fails to provide a good approximation. Therefore, the range $\mathcal{R}$ must be "large". In this case we will show that the intersection number of $A$ must be exponential. We will see that the intersection number serves as a lower bound to the communication complexity of $A$, and so we get that any MIR-approximation algorithm that provides a good approximation ratio must have exponential communication complexity.

The proof of the theorem starts with Subsection 2.3, where the cover number is formally defined and its relation to the approximation ratio is shown. In Subsection 2.4 we define and discuss the second measure: the intersection number. The proof concludes in Subsection 2.5 by showing the relationship between the measures.

\subsection{Complexity Measure I: The Cover Number}

Intuitively we wish to rely on the size of the range. Yet, naive counting will fail to provide good results, since a single allocation in the range may contain many "degenerate allocations". For example, if the range contains an allocation that assigns all items to some bidder $i$, it also contains all allocations such that $i$ is assigned any subset of the items, and the rest of the bidders get nothing. These exponentially many allocations are degenerate in the sense that we can assume that they are not in the range of the algorithm without changing the guaranteed approximation ratio of the $A$. We therefore use an alternative measure for describing the "size" of the range.

Definition 2.2 A set $\mathcal{C}$ of allocations is said to be a cover set of another set of allocations $\mathcal{R}$ if for each $S \in \mathcal{R}$ there exists some $C \in \mathcal{C}$ such that for all $i, S_{i} \subseteq C_{i}$.

The cover number of a set of allocations $\mathcal{R}$ is defined to be the size of the minimum cardinality cover set of $\mathcal{R}$. The cover number is denoted by cover $(\mathcal{R})$.

In the next lemma we prove that if $\operatorname{cover}(\mathcal{R})$ is small, then there exists some instance in which $A$ provides only $\Omega(n)$-approximation.

Lemma 2.3 Let $A$ be an MIR-algorithm with range $\mathcal{R}$. If cover $(\mathcal{R})<e^{\frac{m}{300 n}}$ then there is an instance in which A provides no more than $\frac{1.01}{n}$-fraction of the welfare.

Proof: We randomly construct an instance of a combinatorial auction with additive valuations. Since the valuations are additive, we only need to specify the value of $v_{i}(\{j\})$ for each bidder $i$ and item $j$. This is done in the following way: for each item $j \in M$ choose exactly one bidder, where each bidder is selected with probability of exactly $\frac{1}{n}$. Let $i$ be the selected bidder. We set the value of $v_{i}(\{j\})$ to be 1 . For each $i^{\prime} \neq i$ we set the value of $v_{i^{\prime}}(\{j\})$ to be 0 .

\footnotetext{
${ }^{3}$ We note that extending our results to weighted VCG-based mechanisms is straightforward.
} 
First, observe that the value of the optimal solution in the random instance is exactly $m$. Nevertheless we will see that with non-negative probability the welfare provided by the MIR-algorithm $A$ is only $\frac{1.01}{n} m$. Hence, the approximation ratio provided by $A$ is no better than $\frac{n}{1.01}$. The following version of the Chernoff bounds will be useful.

Claim 2.4 (Chernoff bound) Let $X_{1}, \ldots X_{m}$ be independent random variables that take values in $\{0,1\}$, such that for all $i, \operatorname{Pr}\left[X_{i}=1\right]=p$ for some $p$. Then for every $0 \leq \delta \leq 2 e-1$ it holds that:

$$
\operatorname{Pr}\left[\Sigma_{i} X_{i}>(1+\delta) p m\right] \leq e^{-\frac{p m \delta^{2}}{3}}
$$

Let $\mathcal{C}$ be the minimum cardinality cover set of $\mathcal{R}$ with $|\mathcal{C}|=\operatorname{cover}(\mathcal{R})$. Fix some $C \in \mathcal{C}$. The probability that $v_{i}(\{j\})=1$, and that $j \in C_{i}$ is exactly $\frac{1}{n}$, for any bidder $i$ and item $j$. By the

Chernoff bound, $\operatorname{Pr}\left[\Sigma_{i} v_{i}\left(C_{i}\right)>\frac{1+\delta}{n} m\right] \leq e^{-\frac{\delta^{2} m}{3 n}}$. We now claim, by using the union bound, that if $\operatorname{cover}(\mathcal{R})<e^{\frac{\delta^{2} m}{3 n}}$ then there exists some instance such that no allocation in $\mathcal{C}$ provides a welfare of more than $\frac{1+\delta}{n} m$. Therefore it is obvious that no allocation in $\mathcal{R}$ can provide a welfare of more than $\left(\frac{1+\delta}{n}\right) m$ for this instance. The lemma follows by choosing $\delta=.01$.

\subsection{Complexity Measure II: The Intersection Number}

The second complexity measure we consider is the intersection number. We will show that the intersection number of the range of an MIR algorithm is a lower bound to its communication complexity. Before defining the intersection number, we need a structural definition of a set of allocations.

Definition 2.5 We say that a set of allocations $\mathcal{R}$ is regular if there exist constants $s_{1}, \ldots, s_{n}$ such that for all $S \in \mathcal{R}$ and for all $1 \leq i \leq n$ it holds that $\left|S_{i}\right|=s_{i}$.

We are now ready to define the complexity measure itself.

Definition 2.6 A set of allocations $\mathcal{D}$ is called an $(i, j)$-intersection set if for each $D, D^{\prime} \in \mathcal{D}$, $D \neq D^{\prime}$, it holds that $D_{i} \cap D_{j}^{\prime} \neq \emptyset$.

Define the intersection number of a set of allocations $\mathcal{R}$, denoted by intersect $(\mathcal{R})$, to be the maximum cardinality regular $(i, j)$-intersecting set which is a subset of $\mathcal{R}$, where the maximum is taken over all pairs of bidders $i$ and $j$.

Notice that in the definition of the intersection number we require that the intersection set will be regular.

The next lemma shows that we can use the intersection number as a lower bound to the communication complexity of the algorithm.

Lemma 2.7 Let $A$ be an MIR-algorithm for combinatorial auctions with submodular bidders with range $\mathcal{R}$. Let intersect $(\mathcal{R})=d$. Then, the communication complexity of $A$ is $\Omega(d)$. This result holds even for randomized protocols and for non-deterministic protocols.

Proof: We reduce from the disjointness problem (see [16]). In this problem Alice holds a $d$-bit string $a_{1}, \ldots, a_{d}$, and Bob holds a $d$-bit string $b_{1}, \ldots, b_{d}$. The goal is to decide whether there exists some index $k$ such that $a_{k}=b_{k}=1$. It is known that solving the disjointness problem requires $\Omega(d)$ communication bits, even for nondeterministic and randomized protocols. 
Let $\mathcal{D}=\left\{D^{1}, \ldots, D^{d}\right\}$ be an $(i, j)$-intersection set of $\mathcal{R}$. $\mathcal{D}$ is regular, so for each bidder $t$ there exists a constant $s_{t}$ such that $\left|D_{t}\right|=s_{t}$, for all $D \in \mathcal{D}$. Construct a combinatorial auction with $m$ items in the following way: Alice will play the role of bidder $i$, and Bob will play the role of the rest of the bidders, in particular bidder $j$. We now define the valuations of the bidders. Let the valuation of bidder $i$ played by Alice be:

$$
v_{i}(S)= \begin{cases}|S|, & |S| \leq s_{i}-1 \\ s_{i}, & \exists k \text { s.t. } D_{i}^{k} \subseteq S \text { and } a_{k}=1 \\ s_{i}-2^{-\left(|S|-s_{i}+1\right)}, & \text { otherwise }\end{cases}
$$

The valuation $v_{j}$ is defined in an analogous way. Let the valuations of the rest of the bidders be zero on any bundle. The reader is encouraged to verify that all valuations are indeed submodular.

Observe that if there exists some index $k$ such that the $k$ 'th input bit of both players is 1 , then the optimal welfare is $s_{i}+s_{j}$. Otherwise, the optimal welfare is strictly less than $s_{i}+s_{j}$. To see this notice that if bidder $i$ gains a value of $s_{i}$ from the bundle $S_{1}$ he was assigned by $A$, then there must be an index $k$ such that $D_{i}^{k} \subseteq S_{1}$ and $a_{k}=1$. In order of bidder $j$ to gain a value of $s_{j}$ he must have an index $k^{\prime}$ such that $D_{j}^{k^{\prime}} \subseteq S_{2}$. However, $\mathcal{D}$ is an $(i, j)$-intersection set and so it must hold that $D_{i}^{k} \cap D_{j}^{k^{\prime}} \neq \emptyset$, and thus $S_{1} \cap S_{2} \neq \emptyset$. Clearly, the optimal welfare in this case is less than $s_{i}+s_{j}$.

By construction, if the optimal welfare is $s_{i}+s_{j}$ then it can be achieved by an allocation in $\mathcal{R}$. $A$ is a maximal-in-range algorithm, and so the value of the allocation returned by $A$ in this case must be $s_{i}+s_{j}$. Thus, we will be able to decide if there is a some index $k$ such that $a_{k}=b_{k}=1$. Hence, the communication complexity of $A$ is at least as that of the disjointness problem: $\Omega(d)$.

Notice that our lower bound applies even for computing the value of the optimal allocation in $\mathcal{R}$, and thus applies not only to MIR algorithms but also to algorithms that are equivalent to MIR.

\subsection{The Relationship between the Measures}

It is easy to see that $\operatorname{cover}(\mathcal{R}) \geq \operatorname{inter} \operatorname{sect}(\mathcal{R})$. This subsection shows that the gap between the two is not too large. Specifically, if $\operatorname{inter} \operatorname{sect}(\mathcal{R})$ is small, then $\operatorname{cover}(\mathcal{R})$ is small too.

Lemma 2.8 Let $\mathcal{R}$ be a set of allocations with intersect $(\mathcal{R}) \leq d$. Then

$$
\operatorname{cover}(\mathcal{R})<(8 d)^{m^{\frac{3}{5} n}} \cdot m^{4 m^{\frac{2}{5}} n^{2}}
$$

As a corollary ${ }^{4}$, let $n=m^{\frac{1}{6}}$. If $\operatorname{cover}(\mathcal{R})>e^{\frac{m}{300 n}}$ then $\operatorname{inter} \operatorname{sect}(\mathcal{R}) \geq e^{m \frac{1}{15}}$. Thus, proving the lemma, together with Lemmas 2.3 and 2.7, derives Theorem 2.1.

Proof: (of Lemma 2.8) The lemma will follow from the following claim.

Claim 2.9 Fix some $w, 1 \leq w \leq m$. Let $\widetilde{\mathcal{R}}$ be a regular set of allocations. If inter sect $(\widetilde{\mathcal{R}}) \leq d$ then there is a subset $\mathcal{E}$ of $\widetilde{\mathcal{R}}$ where $\frac{|\overline{\mathcal{E}}|}{|\widetilde{\mathcal{R}}|} \geq(8 d)^{-m n / w} 4^{-n^{2}}$, and cover $(\mathcal{E}) \leq w^{n} m^{w n^{2}}$.

The lemma is proved by partitioning $\mathcal{R}$ to up to $m^{n}$ classes of regular allocations, $\mathcal{R}_{1}, \ldots, \mathcal{R}_{m^{n}}$, one for each possible choice of constants $s_{1}, \ldots, s_{n}$ from Definition 2.5. Each $s_{i}$ is between 1 and $m$, so there are at most $m^{n}$ classes. For each class $\mathcal{R}_{s}$ we will set an upper bound on $\operatorname{cover}\left(\mathcal{R}_{s}\right)$ separately:

Let $\mathcal{E}_{1}^{s}$ be the set obtained from the claim. Look at $\mathcal{R}_{s} \backslash \mathcal{E}_{1}^{s}$, and obtain from the claim another set $\mathcal{E}_{2}^{s} \subseteq \mathcal{R}_{s} \backslash \mathcal{E}_{1}^{s}$ with small cover, and so on. After $(8 d)^{\frac{m n}{w}} \cdot 4^{n^{2}} \cdot \log \left|\mathcal{R}_{s}\right|$ steps $\mathcal{R}_{s}$ is completely

\footnotetext{
${ }^{4}$ The result is actually stronger: fix a constant $\epsilon>0$, and let $n<m^{\frac{1}{5}-\epsilon}$. If $\operatorname{cover}(\mathcal{R})>e^{\frac{m}{300 n}}$ then $\operatorname{intersect}(\mathcal{R}) \geq$ $e^{m^{\epsilon}}$. The statement of the theorem improves accordingly.
} 
covered. Now $\operatorname{cover}\left(\mathcal{R}_{s}\right)$ can be bounded from above by $\Sigma_{k} \operatorname{cover}\left(\mathcal{E}_{k}^{s}\right)$. By bounding from above the size of each class $\left|\mathcal{R}_{s}\right|$ by $|\mathcal{R}| \leq n^{m}$, we have that (by choosing $w=m^{\frac{2}{5}}$ ):

$\operatorname{cover}(\mathcal{R}) \leq \Sigma_{a=1}^{m^{n}} \operatorname{cover}\left(\mathcal{R}_{s}\right) \leq \Sigma_{a=1}^{m^{n}} \Sigma_{k}\left|\mathcal{E}_{k}^{s}\right| \leq m^{n} \cdot(8 d)^{\frac{m n}{w}} \cdot 4^{n^{2}} \cdot m \log n \cdot w^{n} m^{w n^{2}} \leq(8 d)^{m^{\frac{3}{5} n}} \cdot m^{4 m^{\frac{2}{5}} n^{2}}$

Before proving Claim 2.9 itself, and thus Lemma 2.8, we will need some notation.

Definition 2.10 Let $T_{1}, \ldots, T_{n} \subseteq M$. We say that a set of allocations $\mathcal{R}$ is $\left(T_{1}, \ldots, T_{n}\right)$-structured if for all $S \in \mathcal{R}$ it holds that $S_{i} \subseteq T_{i}$.

Definition 2.11 We will say that an allocation $S$ is $w$-(i,j)-aligned in structure $\left(T_{1}, \ldots, T_{n}\right)$, if $\left|S_{i} \cap T_{j}\right| \leq w$. We will omit $w$ when it will be clear from the context.

The idea in proving Claim 2.9 will be to find a large subset $\mathcal{E} \subseteq \widetilde{\mathcal{R}}$, which is "sufficiently" aligned. Next we show that such subset has a small cover number.

Claim 2.12 Let $\mathcal{E}$ be a $T=\left(T_{1}, \ldots, T_{n}\right)$-structured set of allocations. If for each pair of bidders $i$ and $j$ either all allocations in $\mathcal{E}$ are $w$ - $(i, j)$-aligned in $T$ or all allocations in $\mathcal{E}$ are $w$ - $(j, i)$-aligned in $T$, then cover $(\mathcal{E}) \leq w^{n} m^{n^{2} w}$.

Proof: (of Claim 2.12) For each bidder $i$ define a set of bidders $I_{i}$, where bidder $j$ is in $I_{i}$ if all allocations in $\mathcal{E}$ are $(i, j)$-aligned in $T$. Clearly, for each $i$ and $j$, either $j \in I_{i}$ or $i \in I_{j}$. Let $B_{i}=T_{i} \backslash\left(\cup_{j \in I_{i}} T_{j}\right)$. The construction guarantees that $\left(B_{1}, \ldots, B_{n}\right)$ "almost" covers $\mathcal{E}$ in the sense that for bidder $i$ and $S \in \mathcal{E},\left|S_{i} \backslash B_{i}\right| \leq n w$. Also notice that by construction for each two different bidders $i$ and $j, B_{i} \cap B_{j}=\emptyset$. Define the cover $\mathcal{C}$ as follows:

$$
\begin{aligned}
& \mathcal{C}=\left\{P \mid P \text { is an allocation in the form }\left(\left(B_{1} \cup Q_{1}\right) \backslash \cup_{j \neq 1} Q_{j}, \ldots,\left(B_{n} \cup Q_{n}\right) \backslash \cup_{j \neq n} Q_{j}\right),\right. \\
& \left.\quad \text { and for each } i,\left|Q_{i}\right| \leq n w\right\}
\end{aligned}
$$

Observe that since each $\left|Q_{i}\right| \leq n w$ we have that $|\mathcal{C}| \leq\left(\sum_{r=1}^{w} r\left(\begin{array}{c}m \\ n r\end{array}\right)\right)^{n} \leq\left(w(m)^{n w}\right)^{n}=w^{n} m^{n^{2} w}$. Also notice that $\mathcal{C}$ is a cover set of $\mathcal{E}$. To see this, fix an allocation $S \in \mathcal{E}$. For each $i$, let $Q_{i}=S_{i} \backslash B_{i}$. Observe that each $\left|Q_{i}\right| \leq n w$, and that the $Q_{i}$ 's define an allocation that is in $\mathcal{C}$ and covers $S$.

Now we are ready to prove the main claim, and thus finish the proof of Lemma 2.8.

Proof: (of Claim 2.9) The construction of $\mathcal{E}$ will be divided into several steps. During the construction we maintain a sequence of subsets of $\widetilde{\mathcal{R}}: \mathcal{R}_{0}, \mathcal{R}_{1}, \ldots$ and structures $T^{0}, T^{1}, \ldots$, such that each $\mathcal{R}_{t}$ is $T^{t}$-structured. We start by setting $\mathcal{R}_{0}=\widetilde{\mathcal{R}}$ and $T^{0}=(M, \ldots, M)$.

In each step we look at a pair of bidders $i$ and $j$ such that either all allocations in $\mathcal{R}_{t}$ are $(i, j)$ aligned in $T^{t}$ or all allocations in $\mathcal{E}$ are $(j, i)$-aligned in $T^{t}$. If there is no such pair then let $\mathcal{E}=\mathcal{R}_{t}$ and the construction is over. Otherwise, look at all allocations in $\mathcal{R}_{t}$ that are either $(i, j)$-aligned or $(j, i)$-aligned in $T^{t}$. If there are at least $\left|\mathcal{R}_{t}\right| / 2$ such allocations then we set $\mathcal{R}_{t+1}$ to be the largest set of the two: all allocations in $\mathcal{R}_{t}$ that are $(i, j)$-aligned, or all allocations in $\mathcal{R}_{t}$ that are $(j, i)$-aligned. Set the structure $T^{t+1}$ to be $T^{t}$. Notice that $\left|\mathcal{R}_{t+1}\right| \geq\left|\mathcal{R}_{t}\right| / 4$, and that $\mathcal{R}_{t+1}$ is $T^{t+1}$-structured. We call this step an alignment step, and proceed to the next step.

Otherwise, let $\mathcal{R}_{t}^{\prime}$ be the set of allocations in $\mathcal{R}_{t}$ that are neither $(i, j)$-aligned nor $(j, i)$-aligned. Notice that $\left|\mathcal{R}_{t}^{\prime} \geq \frac{\mathcal{R}_{t}}{2}\right|$. Take a maximal $(i, j)$-intersection set $\mathcal{D} \subseteq \mathcal{R}_{t}^{\prime}-$ of size at most $d$. Now for every allocation $S \in \mathcal{R}_{t}^{\prime} \backslash \mathcal{D}$ there exists some $D \in \mathcal{D}$ such that $D_{i} \cap S_{j}=\emptyset$ or $D_{j} \cap S_{i}=\emptyset$. Otherwise we have that $S \in \mathcal{D}$, contradicting the fact that $\mathcal{D}$ is a maximal intersection set. Thus, for some $D \in \mathcal{D}$ we have that for at least $\left(\left|\mathcal{R}_{t}^{\prime}\right|-d\right) /(2 d)$ allocations in $\mathcal{R}_{t}^{\prime}$ either $D_{i} \cap S_{j}=\emptyset$ or 
$D_{j} \cap S_{i}=\emptyset$. Let us assume that for at least $\left(\left|\mathcal{R}_{t}^{\prime}\right|-d\right) /(2 d)$ allocations in $\mathcal{R}_{t}^{\prime}$ the first option occurs. Define $\mathcal{R}_{t+1}$ to be this set of $\left(\left|\mathcal{R}_{t}^{\prime}\right|-d\right) /(2 d) \geq\left|\mathcal{R}_{t}\right| /(8 d)$ allocations. Let $T_{j}^{t+1}=T_{j}^{t} \backslash D_{i}$. Also let $T_{k}^{t+1}=T_{k}^{t}$, for each $k \neq i$. Now notice that since $\mathcal{D}$ is a set of allocations that are not $(i, j)$-aligned in $T_{t}$, we have that $D_{i} \cap T_{j}^{t}>w$. We therefore have that $\left|T_{j}^{t+1}\right|<\left|T_{j}^{t}\right|-w$. (The other case is handled similarly, but this time by shrinking $T_{i}^{t+1}$ rather than $T_{j}^{t+1}$.) By construction we have that $\mathcal{R}_{t+1}$ is $T^{t+1}$-structured. Term this step a shrinkage step, and continue to the next step.

Denote by $l$ the number of steps the process went on. At most $\frac{n m}{w}$ steps are shrinkage steps, since in each shrinkage step $\Sigma_{i}\left|T_{i}^{t}\right|$ loses an additive of at least $w$. In addition, there are at most $\left(\begin{array}{l}n \\ 2\end{array}\right)$ alignment steps, one for each pair of bidders. Therefore $|\mathcal{E}|=\left|\mathcal{R}_{l}\right| \geq \frac{|\widetilde{\mathcal{R}}|}{(8 d)^{m n / w_{4}\left(n^{2}\right)}}$. Also note that in the end of the process for each pair of bidders $i$ and $j$ either all allocations in $\mathcal{E}$ are $(i, j)$-aligned in $T^{l}$ or all allocations in $\mathcal{E}$ are $(j, i)$-aligned in $T^{l}$ (observe that an allocation that became properly aligned after an alignment step will remain so during the rest of the process.) By Claim 2.12 we have that cover $(E) \leq w^{n} m^{n^{2} w}$, and thus Claim 2.9 is proved.

This concludes the proof of Lemma 2.8.

\section{A Characterization of VCG-Based Algorithms}

In [24] it was proved that any VCG-based mechanism for general combinatorial auctions is equivalent to MIR algorithm. We slightly generalize this proof to hold for more settings, including the one considered in this paper.

Let $\mathcal{A}$ be a set of alternatives (in our application, $\mathcal{A}$ will be the set of allocations). For all $i$ let $V_{i} \subseteq R^{\mathcal{A}}$ be a set of valuations on $\mathcal{A}$ and denote $V=V_{1} \times \ldots \times V_{n}$. A mechanism is composed of an allocation rule $f: V \rightarrow \mathcal{A}$ and payment rules $p=\left(p_{1}, \ldots, p_{n}\right)$, where $p_{i}: V \rightarrow \mathbb{R}$.

Definition 3.1 A mechanism $(f, p)$ is called VCG-based if for every $i$ and some $h_{i}: V_{-i} \rightarrow \mathbb{R}$ we have that for all $v, p_{i}(v)=h_{i}\left(v_{-i}\right)-\Sigma_{j \neq i} v_{j}(f(v))$.

Definition 3.2 A mechanism $(f, p)$ is called incentive compatible if for every $v_{i}, v_{i}^{\prime}, v_{-i}$ we have that $v_{i}\left(f\left(v_{i}, v_{-i}\right)-p_{i}\left(v_{i}, v_{-i}\right) \geq v_{i}\left(f\left(v_{i}^{\prime}, v_{-i}\right)-p_{i}\left(v_{i}^{\prime}, v_{-i}\right)\right.\right.$.

Definition 3.3 An allocation rule $f$ is called maximal in its range (MIR) if for every $v, f(v) \in$ $\arg \max _{r \in \mathcal{R}} \Sigma v_{i}(r)$, where $\mathcal{R}=\{f(v) \mid v \in V\}$ is the range of $f$.

Definition 3.4 An allocation rule $f$ is equivalent to an allocation rule $g$ if for all $v, \Sigma_{i} v_{i}(f(v))=$ $\Sigma_{i} v_{i}(g(v))$.

Theorem 3.5 (slight extension of [24]) : Assume that $V$ satisfies Condition 1 and Condition 2 below. If a mechanism $(f, p)$ is VCG-based and incentive compatible then $f$ is equivalent to a MIR allocation rule.

For Condition 1 and Condition 2 we will need notations:

Definition 3.6 $V^{\prime}=\left\{v \in V \mid \forall a \neq b \in A, \Sigma_{i} v_{i}(a) \neq \Sigma_{i} v_{i}(b)\right\}$.

Condition $1 V^{\prime}$ is dense in $V$ (in the usual metric in $R^{\mathcal{A}}$ ).

Definition 3.7 For $a \in \mathcal{A}$ and $v_{i}, z_{i} \in V_{i}$, We say that $z_{i}$ is a-above $v_{i}$ if for every $b \in \mathcal{A}, z_{i}(a)-$ $v_{i}(a) \geq z_{i}(b)-v_{i}(b)$. 
Condition 2 For every $v_{i}, w_{i} \in V_{i}$ there exists $z_{i} \in V_{i}$ that is a-above $v_{i}$ and a-above $w_{i}$.

Before we prove the theorem, let us just look at the two applications, one considered in this paper, and one in [7]:

1. Multi-unit auctions: (see [7] for an exact definition) $\mathcal{A}=\left\{\left(a_{1} \ldots a_{n}\right) \mid \Sigma_{i} a_{i} \leq m\right\}, V_{i}$ is all weakly monotone functions from $1 \ldots m$ to $\mathbb{R}$. Condition 1 is met since $V^{\prime}$ has measure 0 . Condition 2 is met by giving a sufficiently high value $q$ to getting at least $a_{i}$ items.

2. Combinatorial auctions with submodular bidders: $\mathcal{A}$ is the set of all allocations $\left(S_{1}, \ldots, S_{n}\right)$, and $v_{i}$ is the set of submodular valuations. Condition 1 is met since again $V^{\prime}$ has zero measure while $V$ is fully dimensional. Condition 2 is met by defining an additive valuation (which in particular is submodular) that gives a sufficiently high value for each element in $S_{i}$.

Proof: $\quad$ Let us denote $\mathcal{R}^{\prime}=\left\{f(v) \mid v \in V^{\prime}\right\}$. Notice that by definition $\Sigma_{i} v_{i}(a) \neq \Sigma_{i} v_{i}(b)$ for every $v \in V^{\prime}$ and $a \neq b \in \mathcal{R}^{\prime}$ and in particular the argmax is unique. We will follow [24] and first show that over $V^{\prime}, f$ is exactly maximal in the range $\mathcal{R}^{\prime}$. I.e. that for all $v \in V^{\prime}, f(v)=\arg \max _{r \in \mathcal{R}^{\prime}} \Sigma v_{i}(r)$. Let us assume wlog that all $h_{i}=0$. Before proceeding with the proof let us note two simple claims:

Claim 3.8 If $f(w)=a$ and $z_{i}$ is a-above $w_{i}$ then $f\left(z_{i}, w_{-i}\right)=a$.

Proof: Assume to the contrary $f\left(z_{i}, w_{-i}\right)=b \neq a$. Since the VCG mechanism based on $f$ is incentive compatible, we get by looking at a player with valuation $w_{i}$ that $w_{i}(a)+\Sigma_{j \neq i} w_{j}(a) \geq w_{i}(b)+$ $\Sigma_{j \neq i} w_{j}(b)$ while by looking at a player with valuation $z_{i}$ we get $z_{i}(a)+\Sigma_{j \neq i} w_{j}(a) \leq z_{i}(b)+\Sigma_{j \neq i} w_{j}(b)$. Subtracting the two inequalities we get $w_{i}(a)-z_{i}(a) \geq w_{i}(b)-z_{i}(b)$ but notice that the fact that $z_{i}$ is $a$-above $w_{i}$ gives the opposite inequality which means that in fact $w_{i}(a)-z_{i}(a)=w_{i}(b)-z_{i}(b)$. Adding this equality to the second inequality above gives $w_{i}(a)+\Sigma_{j \neq i} w_{j}(a) \leq w_{i}(b)+\Sigma_{j \neq i} w_{j}(b)$, and thus equality holds in contradiction to $w$ being in $V^{\prime}$.

Claim 3.9 If $f\left(v_{i}, v_{-i}\right) \neq a=\arg \max _{c \in \mathcal{R}^{\prime}} \Sigma_{i} v_{i}(c)$ and $z_{i}$ is a-above $v_{i}$ then $f\left(z_{i}, v_{-i}\right) \neq a=$ $\arg \max _{c \in \mathcal{R}^{\prime}} z_{i}(c)+\Sigma_{j \neq i} v_{j}(c)$.

Proof: The fact that $a=\arg \max _{c \in \mathcal{R}^{\prime}} z_{i}(c)+\Sigma_{j \neq i} v_{j}(c)$ is simply since in moving from $v_{i}$ to $z_{i}$, the value of the argument to the argmax increased more for $a$ than for any other $c \in A$. The fact that $f\left(z_{i}, v_{-i}\right) \neq a$ is since otherwise a bidder with valuation $v_{i}$ will benifit from reporting $z_{i}$.

We are now ready to prove that $f$ is exactly maximal in the range $\mathcal{R}^{\prime}$. Assume towards contradiction that for $v, w \in V^{\prime}, f(v)=b \neq a=\arg \max _{c \in \mathcal{R}^{\prime}} \Sigma_{i} v_{i}(c)$, and $f(w)=a$. For every $i$ fix $z_{i}$ that is $a$-above both $v_{i}$ and $w_{i}$ (using Condition 2). Using Claim 3.8 repeatedly, for all $i$, we get that $f(z)=a$ (at every stage $i$ we look at $z_{1} \ldots z_{i-1}, w_{i}, w_{i+1} \ldots w_{n}$ vs $z_{1} \ldots z_{i-1}, z_{i}, w_{i+1} \ldots w_{n}$.) On the other hand, using Claim 3.9 repeatedly we get that $f(y) \neq a$ (at every stage $i$ we look at $z_{1} \ldots z_{i-1}, v_{i}, v_{i+1} \ldots v_{n}$ vs $\left.z_{1} \ldots z_{i-1}, z_{i}, v_{i+1} \ldots v_{n}\right)$. Contradiction.

We now need to handle $V-V^{\prime}$. Due to Condition 2, for every $v \in V-V^{\prime}$ we can find an infinite sequence of points $v^{j} \in V^{\prime}$ such that $v^{j} \rightarrow v$ and for all $j, f\left(v^{j}\right)=a$ for some fixed $a \in \mathcal{R}^{\prime}$. Our equivalent MIR allocation rule $f^{\prime}$ will define $f^{\prime}(v)=a$ (using e.g. the lexicographic first possible $a$ ). It remains to see that $\Sigma_{i} v_{i}(a)=\Sigma_{i} v_{i}(f(v))$. This follows since (1) $\Sigma_{i} v_{i}(a)=\lim _{j \rightarrow \text { inf }} \Sigma_{i} v_{i}^{j}(a)(\operatorname{simply}$ since $\left.v^{j} \rightarrow v\right)$ and (2) $\Sigma_{i} v_{i}(f(v))=\lim _{j \rightarrow \text { inf }} \Sigma_{i} v_{i}^{j}\left(f\left(v^{j}\right)\right)$. This last equality just means the continuity of the function $\Sigma_{i} v_{i}(f(v))$ in $v$ and can be established by looking at $\left|\Sigma_{i} v_{i}(f(v))-\Sigma_{i} w_{i}(f(w))\right|$ which can be bounded by a telescopic sum of $n$ elements of a similar form but with only a single index $i$ with $v_{i} \neq w_{i}$, i.e. $\left|\left(v_{i}(a)+\Sigma_{j \neq i} v_{j}(a)\right)-\left(w_{i}(b)+\Sigma_{j \neq i} v_{j}(b)\right)\right|$, where $a=f\left(v_{i}, v_{-i}\right)$ and $b=f\left(w_{i}, v_{-i}\right)$. 
This last difference can be bounded by $\max \left(v_{i}(a)-w_{i}(a), v_{i}(b)-w_{i}(b)\right)$ since otherwise a player with valuation $v_{i}$ would rather declare $w_{i}$ (in case the LHS of the difference is smaller), or vice versa (otherwise).

\section{Acknowledgements}

We thank Liad Blumrosen and Nikhil Devanur for pointing out that the algorithms of [3] and [2] are maximal in range. We also thank Liad Blumrosen for comments on an earlier draft of this paper.

\section{References}

[1] A. Archer, C. Papadimitriou, K. Talwar, and E. Tardos. An approximate truthful mechanism for combinatorial auctions with single parameter agent. In Proceedings of the 14th Annual ACM Symposium on Discrete Algorithms (SODA), 2003.

[2] Sanjeev Arora. Polynomial time approximation schemes for Euclidean traveling salesman and other geometric problems. Journal of the ACM, 45(5):753-782, 1998.

[3] Moshe Babaioff and Liad Blumrosen. Computationally-feasible auctions for convex bundles. In 7th. International Workshop on Approximation Algorithms for Combinatorial Optimization Problems (APPROX). LNCS Vol. 3122., pages 27-38, 2004.

[4] Yair Bartal, Rica Gonen, and Noam Nisan. Incentive compatible multi unit combinatorial auctions. In TARK 03, 2003.

[5] Liad Blumrosen and Shahar Dobzinski. Welfare maximization in congestion games. Working Paper. Preliminary version in EC'06.

[6] E. H. Clarke. Multipart pricing of public goods. Public Choice, pages 17-33, 1971.

[7] Shahar Dobzinski and Noam Nisan. Mechanisms for multi-unit auctions. 2006. Working Paper. Available from http://www.cs.huji.ac.il/ shahard.

[8] Shahar Dobzinski, Noam Nisan, and Michael Schapira. Truthful randomized mechanisms for combinatorial auctions. In STOC'06.

[9] Shahar Dobzinski, Noam Nisan, and Michael Schapira. Approximation algorithms for combinatorial auctionss with complement-free bidders. In The 37th ACM symposium on theory of computing (STOC), 2005.

[10] Shahar Dobzinski and Michael Schapira. An improved approximation algorithm for combinatorial auctions with submodular bidders. In SODA'06.

[11] Uriel Feige. On maximizing welfare where the utility functions are subadditive. In STOC'06.

[12] Uriel Feige and Jan Vondrak. Approximation algorithms for allocation problems: Improving the factor of 1-1/e. In FOCS'06.

[13] T. Groves. Incentives in teams. Econometrica, pages 617-631, 1973.

[14] Ron Holzman, Noa Kfir-Dahav, Dov Monderer, and Moshe Tennenholtz. Bundling equilibrium in combinatrial auctions. Games and Economic Behavior, 47:104-123, 2004. 
[15] Subhash Khot, Richard J. Lipton, Evangelos Markakis, and Aranyak Mehta. Inapproximability results for combinatorial auctions with submodular utility functions. In WINE'05, 2005.

[16] Eyal Kushilevitz and Noam Nisan. Communication Complexity. Cambridge University Press, 1997.

[17] Ron Lavi, Ahuva Mu'alem, and Noam Nisan. Towards a characterization of truthful combinatorial auctions. In The 44th Annual IEEE Symposium on Foundations of Computer Science (FOCS), 2003.

[18] Ron Lavi and Chaitanya Swamy. Truthful and near-optimal mechanism design via linear programming. In FOCS 2005.

[19] Benny Lehmann, Daniel Lehmann, and Noam Nisan. Combinatorial auctions with decreasing marginal utilities. In ACM conference on electronic commerce, 2001.

[20] Daniel Lehmann, Liadan Ita O'Callaghan, and Yoav Shoham. Truth revelation in approximately efficient combinatorial auctions. In JACM 49(5), pages 577-602, Sept. 2002.

[21] A. Mas-Collel, W. Whinston, and J. Green. Microeconomic Theory. Oxford university press, 1995.

[22] Ahuva Mu'alem and Noam Nisan. Truthful approximation mechanisms for restricted combinatorial auctions. In $A A A I-02,2002$.

[23] Noam Nisan. The communication complexity of approximate set packing and covering. In ICALP 2002.

[24] Noam Nisan and Amir Ronen. Computationally feasible vcg-based mechanisms. In ACM Conference on Electronic Commerce, 2000.

[25] Noam Nisan and Amir Ronen. Algorithmic mechanism design. Games and Economic Behaviour, 35:166 - 196, 2001. A preliminary version appeared in STOC 1999.

[26] Noam Nisan and Ilya Segal. The communication requirements of efficient allocations and supporting prices, 2006. In the Journal of Economic Theory.

[27] Kevin Roberts. The characterization of implementable choise rules. In Jean-Jacques Laffont, editor, Aggregation and Revelation of Preferences. Papers presented at the first European Summer Workshop of the Economic Society, pages 321-349. North-Holland, 1979.

[28] Vijay V. Vazirani. Approximation algorithms. Springer-Verlag New York, Inc., New York, NY, USA, 2001.

[29] W. Vickrey. Counterspeculation, auctions and competitive sealed tenders. Journal of Finance, pages 8-37, 1961. 